\title{
Outcomes of the Adams-Berger Ligament Reconstruction for the Distal Radioulnar Joint Instability in 95 Consecutive Cases
}

\author{
Joshua A. Gillis, MD ${ }^{1, *}$ Endre Soreide, MD ${ }^{1,2, *}$ Joseph S. Khouri, MD ${ }^{1}$ Assaf Kadar, MD ${ }^{3,4}$ \\ Richard A. Berger, MD, PhD ${ }^{1}$ Steven L. Moran, $\mathrm{MD}^{1,3}$
}

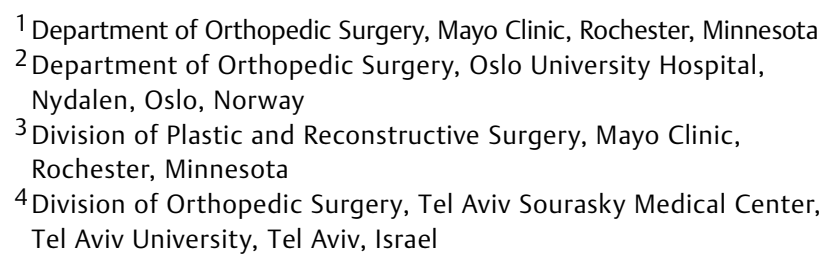

J Wrist Surg 2019;8:268-275.
Address for correspondence Steven L. Moran, MD, Division of Plastic and Reconstructive Surgery, Mayo Clinic, 200 First Street SW, Rochester, MN 55905 (e-mail: moran.steven@mayo.edu).

\begin{abstract}
Keywords

- Adams-Berger procedure

- DRUJ instability

- distal radial ulnar joint

- DRUJ reconstruction

- ulnar-sided wrist pain

Objective The objective of this article is to evaluate the outcomes and complication rate for Adams-Berger anatomic reconstruction of the distal radioulnar joint (DRUJ), in addition, to determine the role of sigmoid notch anatomy on failure.

Methods We conducted a retrospective chart review to evaluate adult patients that had undergone reconstruction of the DRUJ for instability with the Adams-Berger procedure between 1998 and 2015 within our institution with $>24$ months follow-up. Charts were reviewed for patient demographics, mechanism of injury, outcome, and complications.

Results Ninety-five wrists in 93 patients were included. Mean age at surgery was 37.3 years with 65.2 months follow-up. At the last follow-up, $90.8 \%$ had a stable DRUJ, 5.3\% did not, and $3.4 \%$ had some laxity. Postoperatively, $75.9 \%$ described either no pain or mild pain. Grip strength increased while pronosupination decreased. Procedure success was $86.3 \%$, as 12 patients underwent revision at 13.3 months postoperatively. Reconstructive failure was more common in females when an interference screw was utilized for tendon fixation. Age, timing of surgery, type of graft, sigmoid notch anatomy, and previous surgery did not affect revision or failure rate.

Conclusion Our findings demonstrate that Adams-Berger reconstruction of the DRU] provides reliable long-term results with an overall success of $86 \%$ at $>5$ years followup.

Level of evidence/Type of study This is a Level IV, therapeutic study.
\end{abstract}

Distal radioulnar joint (DRUJ) instability can lead to wrist pain and loss of hand function due to reduced forearm rotation and decreased grip strength. ${ }^{1,2}$ Stability of the

\footnotetext{
These authors are co-authors as they contributed equally to this article.
}

received

December 6, 2018

accepted after revision

February 27, 2019

published online

April 22, 2019
DRUJ is provided primarily by the dorsal and volar radioulnar ligaments of the triangular fibrocartilage complex (TFCC). ${ }^{3}$ If both the palmar and dorsal ligaments are disrupted, it results into gross instability of the DRUJ. ${ }^{4}$ If these ligaments heal improperly following injury to the TFCC or are damaged beyond repair, then DRUJ reconstruction is indicated.
Copyright $\odot 2019$ by Thieme Medical Publishers, Inc., 333 Seventh Avenue, New York, NY 10001, USA Tel: +1(212) 584-4662.
DOI https://doi.org/ 10.1055/s-0039-1685235. ISSN 2163-3916. 
For most acute cases, arthroscopic or open surgical repair of the TFCC can restore stability caused by peripheral tears or foveal disruption of the deep fibers of the radioulnar ligaments. ${ }^{5,6}$ However, in chronic injuries where anatomic restoration of the TFCC is not possible, or in cases which have failed previous attempts at repair, DRUJ reconstruction with tendon grafts may be indicated.

Adams and Berger described the anatomic reconstruction of the distal radioulnar ligaments using a tendon graft in $2002 .^{1,7}$ The Adams-Berger procedure aims to restore kinematics and function of the DRUJ by reconstructing the dorsal and volar radial ulnar ligaments with a tendon graft. It is indicated in patients with symptomatic instability of the DRUJ due to irreparable TFCC lesions or recurrent instability after primary repair or stabilizing procedures without degenerative changes. ${ }^{8-10}$ There are few published series examining the long-term outcomes, risk factors for failure, and complications of this procedure. The aim of our study was to assess the long-term outcomes and revision rate in patients undergoing Adams-Berger anatomic ligament reconstruction of the DRUJ at our institution between 1998 and 2015 and the effect of sigmoid notch anatomy and graft fixation type on reconstructive success.

\section{Materials and Methods}

Following institutional review board approval, we retrospectively identified adult patients who had undergone anatomic reconstruction of the DRUJ by the two senior authors at our institution between 1998 and 2015 with $>24$ months followup. Strengthening the Reporting of Observational Studies in Epidemiology (STROBE) guidelines were used in the preparation of this study. Charts were reviewed for patient demographics, mechanism and timing of injury, presurgical physical findings, indication and timing of surgery, and complications. In addition, we obtained clinical follow-up data with special emphasis on revision surgery and functional outcomes during the last noted clinical follow-up. This included pain relief (patient reported as none, mild, moderate, or severe), correction of instability, range of motion (ROM), grip strength, and Mayo Wrist Score (MMWS). Grip strength was corrected for right-hand dominance, assuming it is $15 \%$ stronger, while left handed patients did not have any correction and the two patients with bilateral surgery were excluded from grip strength analysis. Computed tomographic scans were performed to determine sigmoid notch anatomy, as defined by Tolat et al. ${ }^{11}$ A procedure was considered a failure if the patient went on to revision surgery, or had recurrent instability. A mechanical failure occurred when the patient heard a "pop" prior to the development of recurrent instability or if the magnetic resonance imaging showed disruption of the graft.

\section{Assessment of DRU] Instability}

All patients had an exam of the hand, wrist, forearm, and specific examinations for DRUJ instability by one of the senior authors. ROM was measured with a goniometer and grip strength with a calibrated JAMAR dynamometer. Based on the clinical and radiographic evaluation of DRUJ stability, patients with symptomatic instability and without radiographic evidence of degenerative changes underwent diagnostic arthroscopy to assess the DRUJ, TFCC, and the radiocarpal joints. Arthroscopic verification of DRUJ instability coupled with an irreparable TFCC lesion in patients without degenerative changes in the sigmoid notch and ulnar head was an indication for open anatomic DRUJ reconstruction.

\section{Surgical Technique}

A 4-cm dorsal skin incision between the fifth and sixth extensor compartments was made. The fifth compartment was opened and the extensor digiti quinti (EDQ) tendon was retracted. The DRUJ was exposed with an L-shaped capsular incision along the dorsal rim of the sigmoid notch, parallel to the native location of the dorsal radioulnar ligament. The DRUJ and TFCC were then evaluated.

To determine the distal radius bone tunnel position, a guidewire was placed under fluoroscopic guidance 4 to $5 \mathrm{~mm}$ proximal to the lunate fossa and radial to the sigmoid. A 3-cm palmar skin incision was made between the ulnar neurovascular bundle and digital flexor tendons proximal to the proximal wrist crease. The flexor tendons were retracted radially and the neurovascular bundle ulnarly to identify the volar aspect of the bone tunnel. The guidewire was then overdrilled using a cannulated 3 to $3.5 \mathrm{~mm}$ drill bit and an awl was used to widen the tunnel to allow graft passage. Using the same cannulated drilling technique, a second oblique bone tunnel was made in the distal ulna by drilling retrograde from the fovea to the ulnar aspect of the ulnar shaft. A suture retriever was then passed through the radial tunnel from dorsal to volar and a limb of either an autologous or cadaveric tendon graft was passed dorsally. Choice of tendon (allo vs. autograft) was left to the discretion of the surgeon. The palmaris longus tendon can be of inadequate length for the conventional method of a half-hitch, and thus an allograft may be beneficial. A hemostat was directed from dorsal to volar just distal to the ulnar head and proximal to any remnants of the TFCC and pushed through the volar DRUJ capsule to grasp the palmar limb of the graft. The graft was pulled dorsally and tightened, ensuring not to trap any volar structures.

Both limbs of the graft were passed through the ulnar tunnel. One limb was passed underneath the extensor carpi ulnaris sheath, while the other limb was passed in the opposite direction, with attention not to entrap the ulnar neurovascular bundle. With manual compression applied to a neutral-positioned DRUJ, a half-hitch was made with the two limbs of the graft, pulled tight and then secured with nonresorbable sutures while maintaining graft tension (-Fig. 1). The dorsal DRUJ capsule and extensor retinaculum were closed in layers to add additional stability. To add further stability, the tendon graft may be incorporated into the capsular repair. The EDQ was left transposed and bowstringing was prevented by keeping the distal part of the extensor retinaculum intact.

If an interference screw was preferred for graft fixation, the DRUJ was manually compressed in neutral position and both limbs of the graft were pulled tight at the 

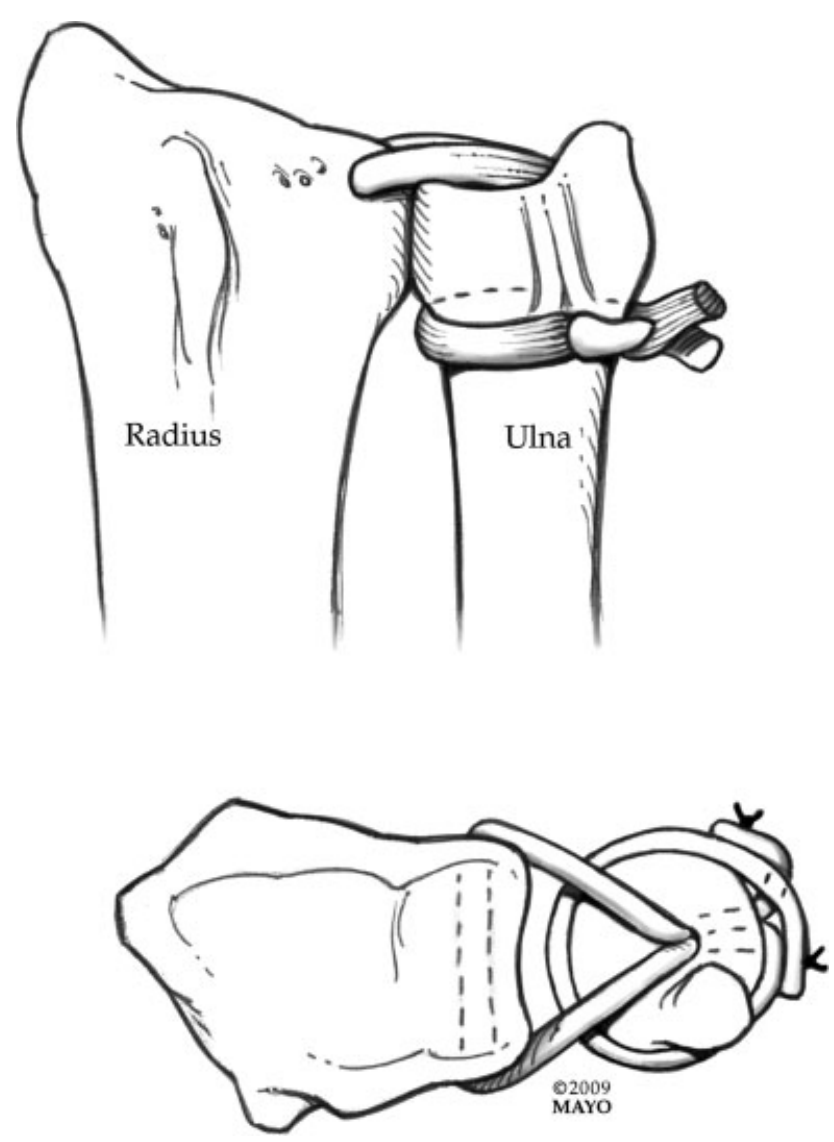

Fig. 1 Anatomical depiction of the conventional loop method of graft fixation of the Adams-Berger technique for anatomical reconstruction of the distal radioulnar ligaments.

proximal opening of the ulnar bone tunnel. The interference screw was advanced in a proximal to distal direction, providing a solid fixation of the graft in the bone tunnel. If bone-anchored sutures were used, two anchors were inserted just proximal to the ulnar tunnel and horizontal mattress sutures were used to secure the graft after a half hitch of the two limbs. Pinning of the ulna to the radius is optional and is independent of the method of graft fixation. In our opinion, pinning should not be necessary following closure of the capsule but could be considered if residual instability is present or due to concerns regarding patient compliance.

Patients were placed into a sugar tong splint for 2 weeks followed by a Muenster cast for 4 weeks. Therapy began at week 6 with gentle forearm ROM exercises. Patients were provided with a clam shell splint for 4 additional weeks as they continued with strengthening therapy and proprioceptive feedback therapy.

\section{Statistical Analysis}

Continuous variables and categorical variables were compared using nonparametric tests. All tests were two sided. Graft success was calculated using the Kaplan-Meyer method and distributional differences compared using log rank test. Statistical significance level was set to 0.05 . We calculated $80 \%$ power to detect a hazard ratio of $\geq 5.1$.

\section{Results}

Our search revealed 95 wrists in 93 patients during the study period. Two female patients had undergone bilateral procedures. Mean patient age at surgery was 37.2 years and mean follow-up time was 65.2 months (range: 28-190 months, median: 38.5 months). Time from injury to surgery was 28.7 months (range: 2-473 months, median: 11.5 months). Preoperative parameters can be seen in - Tables $\mathbf{1}$ to $\mathbf{3}$. Of note, $39 \%$ of the patients had undergone other surgery directed at their wrist symptoms at the time of referral (-Tables 1 and 2). There were 30 "flat" (32.6\%), 8 "s-shaped" (8.7\%), 39 "c-shaped" (42.4\%), and 15 "ski-slope" (16.3\%) shaped sigmoid notches.

Concomitant injuries occurred in 25\% of patients: 9 patients (9.5\%) with a distal radius fracture, 3 patients (3.2\%) with a distal ulnar fracture, 3 patients (3.2\%) with a carpal ligament injury, and 2 patients $(2.1 \%)$ with a forearm fracture. Four of the distal radius fractures and one ulnar styloid fracture underwent open reduction and internal fixation prior to DRUJ reconstruction.

Autograft was used in 42 reconstructions, and allograft (not used until 2002) was used in 53 reconstructions (-Table 3). The majority of grafts were fixated by suturing the tendon graft to itself after looping it circumferentially around the ulnar neck ( - Fig. 1). There were no differences in use of autograft or allograft between male and females, handedness or type of graft fixation (-Table 3 ).

Overall, graft success was $86.3 \%$ (those patients with a stable DRUJ or did not go on to revision surgery) (-Fig. 2).

Table 1 Preoperative parameters of patients undergoing Adams-Berger distal radioulnar joint reconstruction

\begin{tabular}{|l|l|}
\hline Mechanism of injury & $\boldsymbol{n}=\mathbf{8 6}$ \\
\hline Fall on outstretched arm & $38(44 \%)$ \\
\hline Rotatory trauma & $15(17 \%)$ \\
\hline Heavy lifting & $8(9 \%)$ \\
\hline Direct ulnar trauma & $8(9 \%)$ \\
\hline Distraction & $3(3 \%)$ \\
\hline Hyperextension & $4(5 \%)$ \\
\hline Other & $10(12 \%)$ \\
\hline Presenting symptoms & $\mathbf{n}=91$ \\
\hline Ulnar-sided pain & $72(79 \%)$ \\
\hline Global wrist pain & $9(10 \%)$ \\
\hline Instability & $7(8 \%)$ \\
\hline Weakness & $2(2 \%)$ \\
\hline Clunk & $1(1 \%)$ \\
\hline $\begin{array}{l}\text { Number of surgeries prior to } \\
\text { Adams-Berger reconstruction }\end{array}$ & $\mathbf{n}=95$ \\
\hline 0 & $58(61 \%)$ \\
\hline 1 & $25(26 \%)$ \\
\hline 2 & $11(12 \%)$ \\
\hline 3 & $1(1 \%)$ \\
\hline
\end{tabular}


Table 2 Surgeries performed prior to distal radioulnar joint reconstruction in our series of 95 wrists

\begin{tabular}{|l|l|}
\hline Surgery prior to DRUJ reconstruction & Number \\
\hline TFCC repair & 21 \\
\hline TFCC debridement & 9 \\
\hline Distal radius ORIF & 4 \\
\hline Brunelli SL ligament recon & 3 \\
\hline UT split tear suture repair & 3 \\
\hline ECU subsheath repair & 2 \\
\hline Ulnar styloid fracture ORIF & 2 \\
\hline Ulnar styloid nonunion removal & 2 \\
\hline Four-corner fusion & 1 \\
\hline Distal radius corrective osteotomy & 1 \\
\hline LT fusion & 1 \\
\hline Olecranon ORIF & 1 \\
\hline LT ligament repair & 1 \\
\hline LT dislocation CRPP & 1 \\
\hline Pisiform excision & 1 \\
\hline Guyon's canal release & 1 \\
\hline ECU subsheath synovectomy & 1 \\
\hline Ulnar shortening osteotomy & 1 \\
\hline Total & 56 \\
\hline
\end{tabular}

Abbreviations: CRPP, closed reduction and percutaneous pinning; DRU], distal radioulnar joint; ECU, extensor carpi ulnaris; LT, lunotriquetral; ORIF, open reduction and internal fixation; SL, scapholunate; UT, ulnotriquetral; TFCC, triangular fibrocartilage complex.

Eleven females and one male patient underwent revision of the DRUJ reconstruction. There were six mechanical failures and six procedural failures. Time to revision from primary reconstructive surgery was 13.3 months on average (7.6 months, 4-32 months, median, 10.50 months). Success

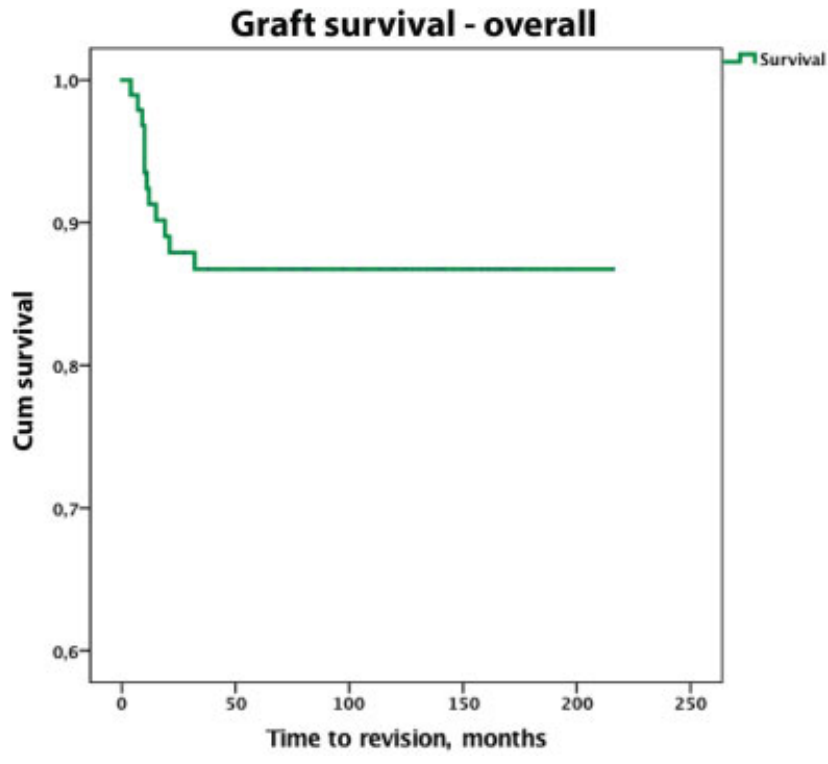

Fig. 2 Kaplan-Meier curve of the overall procedure success utilizing the Adams-Berger technique for distal radioulnar joint reconstruction in 95 wrists.

was significantly better in males compared to females (-Fig. 3 and -Table 4). There was a significant association between the fixation type and the risk of revision $(p<0.001)$. The 1 -year revision free survival was $94 \%$ in the 83 patients with a standard fixation, $75 \%$ in the 8 anchor fixation patients, and $67 \%$ in 4 screw fixation patients (-Fig. 4 and -Table 4). There was no difference in success between the tendon loop and suture anchor.

The pre- and postoperative ROM and grip strength can be seen in -Table 5. Grip strength was statistically higher postoperatively. Pre- and postoperative MMWS data can be seen in - Table 6 . The majority of patients were able to return to work after surgery (- Table 6 ). At the last follow-up, 90.8\% of patients had a stable DRUJ, $5.3 \%$ did not, and $3.4 \%$ had some laxity. At last clinical follow-up, 27.5 and $48.4 \%$ of patients

Table 3 Overview of the use of grafts for the Adams-Berger reconstruction

\begin{tabular}{|c|c|c|c|c|c|}
\hline & & Autograft $(n=42)$ & Allograft $(n=53)$ & Total & $p$-Value $^{a}$ \\
\hline \multirow[t]{2}{*}{ Sex } & Female & $27(44 \%)$ & $35(56 \%)$ & 62 & \multirow[t]{2}{*}{0.859} \\
\hline & Male & $15(45 \%)$ & $18(55 \%)$ & 33 & \\
\hline \multirow{5}{*}{$\begin{array}{l}\text { Graft } \\
\text { source }\end{array}$} & Palmaris longus & $34(87 \%)$ & $5(13 \%)$ & 39 & \multirow[t]{5}{*}{$<0.05^{\mathrm{b}}$} \\
\hline & Plantaris & $7(17 \%)$ & $35(83 \%)$ & 42 & \\
\hline & Gracilis & 0 & $12(100 \%)$ & 12 & \\
\hline & Triceps surae & $1(100 \%)$ & 0 & 1 & \\
\hline & Semitendinosus & 0 & $1(100 \%)$ & 1 & \\
\hline \multirow[t]{3}{*}{$\begin{array}{l}\text { Fixation } \\
\text { of graft }\end{array}$} & $\begin{array}{l}\text { Circumferential around ulnar } \\
\text { neck and sutured to itself }\end{array}$ & $39(47 \%)$ & $44(53 \%)$ & 83 & \multirow[t]{3}{*}{$0.664^{c}$} \\
\hline & Suture anchors & $3(38 \%)$ & $5(62 \%)$ & 8 & \\
\hline & Interference screw & $1(25 \%)$ & $3(75 \%)$ & 4 & \\
\hline
\end{tabular}

${ }^{a}$ Kruskal-Wallis nonparametric test comparing auto- and allograft distribution and sex, graft type, and fixation of graft.

${ }^{b}$ Subgroup analysis demonstrated there were more palmaris longus tendons in the autograft group and more plantaris in the allograft group.

${ }^{C}$ Equal distribution of allo- and autograft compared to graft fixation type. 


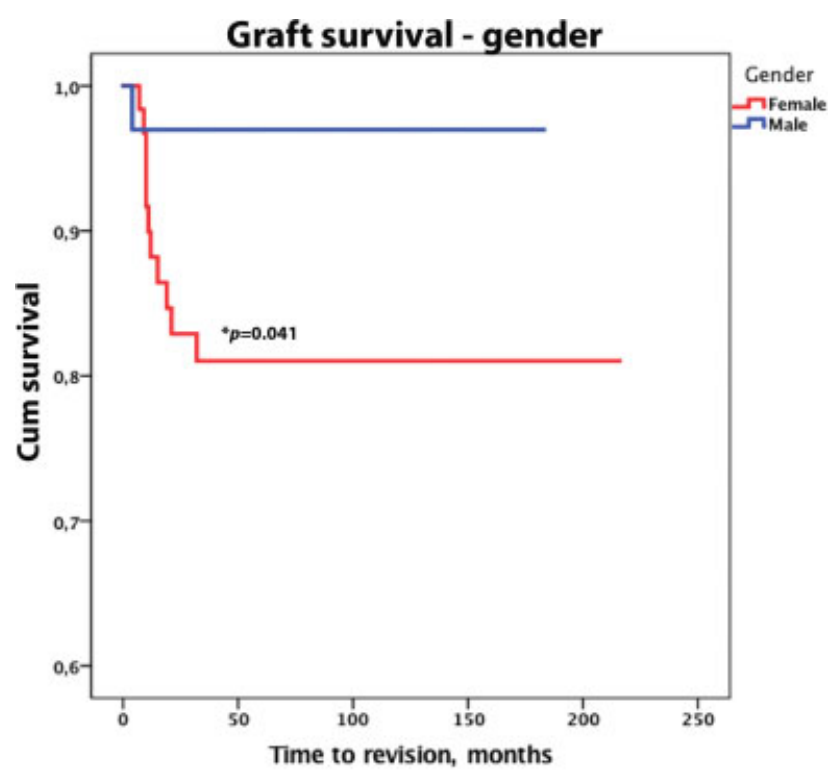

Fig. 3 Kaplan-Meier curve of the procedure success based on sex in 95 wrists that underwent the Adams-Berger technique for distal radioulnar joint reconstruction. described either no pain or mild pain, respectively, with $22.0 \%$ of patients with moderate pain and no patients with severe pain. This was a significant improvement in reported pain $(p<0.05)$.

Complications occurred in $31.6 \%$ of patients (-Table $\mathbf{7}$ ). Six patients suffered a neuropraxia of the ulnar nerve and one patient had a neuroma excised for continued pain. Causes for revision surgery were persistent symptomatic instability of the DRUJ $(n=3)$, recurrent instability of the DRUJ $(n=5)$, and painful degenerative arthritis of the DRUJ demanding surgical intervention $(n=4)$. ROM and grip strength preoperatively and at last follow-up for revision patients can be seen in - Table 8. In eight of the initial revision surgeries, the reconstruction was found to be intact and in two patients the tendon reconstruction had ruptured. One was ruptured at the exit of the ulnar tunnel and one had ruptured volarly. Of those with persistent pain, two were found to have a ulnotriquetral split tear that was treated surgically and one patient had an ulnar styloid nonunion.

Of the 12 revised patients, 6 patients had received surgical treatment prior to the primary Adams-Berger procedure. Allografts were used in 7 of the 12 revised patients at the

Table 4 Overview of graft success based on potential risk factors with hazard ratios for specific subgroups risk of graft failure

\begin{tabular}{|c|c|c|}
\hline Factor & $p$-Value ${ }^{a}$ & Hazard ratio ${ }^{b}$ \\
\hline Gender & 0.041 & \\
\hline Male & & 1.0 (reference) \\
\hline Female & & $6.394(0.785-52.063)$ \\
\hline Reconstruction within or after 6 months & 0.254 & \\
\hline Reconstruction within or after 12 months & 0.436 & \\
\hline Age at time of surgery older or younger than 40 years & 0.091 & \\
\hline Autograft or allograft used for the reconstruction & 0.739 & \\
\hline Autograft & & 1.0 (reference) \\
\hline Allograft & & $1.223(0.373-4.014)$ \\
\hline Involvement of dominant wrist or not & 0.746 & \\
\hline Yes & & $1.191(0.413-3.435)$ \\
\hline No & & 1.0 (reference) \\
\hline Prior surgery to the affected wrist or not & 0.378 & \\
\hline Type of graft fixation & $<0.05$ & \\
\hline Standard loop & (reference) & 1.0 (reference) \\
\hline Bone-anchored suture & 0.194 & $2.769(0.595-12.886)$ \\
\hline Interference screw & $<0.05$ & $9.531(4.155-21.864)$ \\
\hline Concomitant injury & 0.893 & \\
\hline Yes & & $0.914(0.246-3.400)$ \\
\hline No & & 1.0 (reference) \\
\hline Sigmoid notch type & 0.690 & \\
\hline Flat & & $2.150(0.622-7.433)$ \\
\hline S-shaped & & $1.773(0.187-16.847)$ \\
\hline Ski-slope & & $1.730(0.284-10.536)$ \\
\hline C-shaped & (reference) & 1.0 (reference) \\
\hline
\end{tabular}

${ }^{\mathrm{a}}$ Log rank test.

bUnivariate cox models for hazard ratios given. 
Adam-Berger DRUJ Reconstruction Outcomes Gillis et al. 273

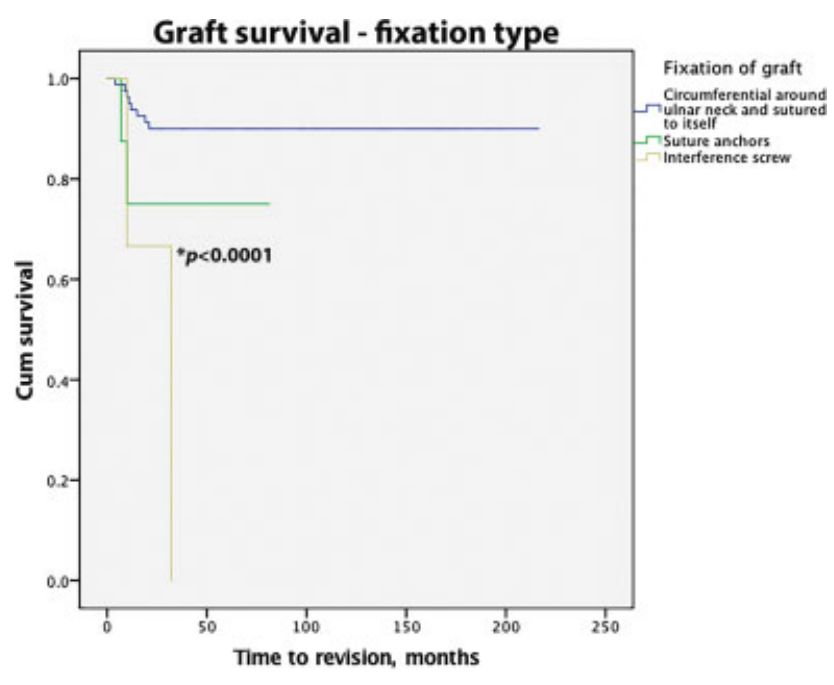

Fig. 4 Kaplan-Meier curve of the procedure success based on graft fixation technique in 95 wrists that underwent the Adams-Berger procedure for distal radioulnar joint reconstruction.

time of original surgery. This included both plantaris tendons $(n=6)$ and a gracilis tendon $(n=1)$. Palmaris longus autografts were the preferred graft for the remaining revised patients $(n=5)$. Four patients underwent ulnar head replacement due to progressive degenerative disease of the DRUJ and two patients had an ulnar head resection.

\section{Discussion}

Different methods of DRUJ reconstruction have been described: a direct extrinsic radioulnar tether, extensor retinaculum capsulorrhaphy, and capsular plication; and an indirect radioulnar link using an ulnocarpal sling or tenodesis (Linscheid-Hui procedure), dynamic muscle transfer, and sigmoid notch reconstruction. ${ }^{2,12-16}$ These procedures do not restore the normal anatomy of the joint and thus may be unreliable and restrict joint motion. ${ }^{14}$ The AdamsBerger reconstruction of the DRUJ aims to link the distal radius and ulna using a tendon graft which mimics the attachments of the native TFCC anatomy. While there are inherent problems of reproducing the elastic modulus of
Table 6 Clinical outcomes at last follow-up

\begin{tabular}{|l|l|}
\hline Pain preoperative & $\boldsymbol{n}=95$ \\
\hline No pain & $0(0.0 \%)$ \\
\hline Mild & $1(1.1 \%)$ \\
\hline Moderate & $62(65.3 \%)$ \\
\hline Severe & $32(33.7 \%)$ \\
\hline Pain at last follow-up ${ }^{\text {a }}$ & $\boldsymbol{n}=91$ \\
\hline No pain & $25(27.5 \%)$ \\
\hline Mild & $44(48.4 \%)$ \\
\hline Moderate & $20(22.0 \%)$ \\
\hline Severe & $0(0.0 \%)$ \\
\hline Stable DRUJ at last follow-up & $\boldsymbol{n}=87$ \\
\hline Yes & $79(90.8 \%)$ \\
\hline No & $5(5.7 \%)$ \\
\hline Some laxity & $3(3.4 \%)$ \\
\hline Work ability preoperative & $\boldsymbol{n}=77$ \\
\hline Unable due to pain & $11(12.14 \%)$ \\
\hline Regular job & $51(57.3 \%)$ \\
\hline Restricted job & $9(10.1 \%)$ \\
\hline Able, but unemployed or student & $6(6.7 \%)$ \\
\hline Work ability at last follow-up & $\boldsymbol{n}=86$ \\
\hline Unable due to pain & $17(18.1 \%)$ \\
\hline Regular job & $40(42.6 \%)$ \\
\hline Restricted job & $28(29.8 \%)$ \\
\hline Able, but unemployed & $1(1.1 \%)$ \\
\hline Patient-reported & $68.9(15.0 \mathrm{SD})^{\mathrm{b}}$ \\
\hline questionnaires & $8.1(2.5 \mathrm{SD})$ \\
\hline Preoperative MMWS ( $=68)$ & $59.6(18.4 \mathrm{SD})$ \\
\hline Postoperative MMWS (n=68) & \\
\hline Satisfaction (VAS) ( $=40)$ & \\
\hline
\end{tabular}

Abbreviations: DRU], distal radioulnar joint; MMWS, modified mayo wrist score; VAS, visual analogue scale.

${ }^{a}$ Wilcoxon signed rank test significant decrease in pain $(p<0.05)$.

bWilcoxon signed rank test significant increase in MMWS $(p<0.05)$.

Table 5 ROM in all patients undergoing Adams-Berger procedure

\begin{tabular}{|c|c|c|c|c|c|c|c|c|c|}
\hline & \multicolumn{3}{|c|}{$\begin{array}{l}\text { Preoperative ROM } \\
\text { (degrees) }\end{array}$} & \multirow[t]{2}{*}{$\%$ of normal } & \multicolumn{3}{|c|}{$\begin{array}{l}\text { Last clinical follow-up, } \\
\text { ROM (degrees) }\end{array}$} & \multirow[t]{2}{*}{$\%$ of normal } & \multirow[b]{2}{*}{$p$-Value ${ }^{a}$} \\
\hline & $n$ & Mean & SD & & $n$ & Mean & SD & & \\
\hline Flexion & 87 & 53.9 & 16.0 & 81.2 & 84 & 52.1 & 16.1 & 78.0 & 0.493 \\
\hline Extension & 87 & 57.0 & 16.5 & 84.7 & 84 & 58.6 & 12.7 & 88.7 & 0.909 \\
\hline Radial deviation & 82 & 18.3 & 6.1 & 84.1 & 63 & 19.8 & 5.4 & 89.9 & 0.340 \\
\hline Ulnar deviation & 81 & 27.2 & 9.2 & 84.1 & 63 & 28.6 & 9.4 & 84.7 & 0.601 \\
\hline Pronation & 86 & 76.9 & 14.7 & 93.8 & 83 & 71.3 & 14.3 & 88.8 & $<0.05$ \\
\hline Supination & 86 & 69.4 & 20.9 & 86.1 & 84 & 62.7 & 20.6 & 77.6 & $<0.05$ \\
\hline Grip strength $(\mathrm{kg})$ & 73 & 21.7 & 13.4 & 68.5 & 71 & 24.2 & 13.9 & 77.4 & $<0.05$ \\
\hline
\end{tabular}

Abbreviation: ROM, range of motion; SD, standard deviation.

${ }^{a}$ Wilcoxon signed-rank test. 
Table 7 Postoperative complications of the anatomic distal radioulnar joint reconstruction in our series of 95 wrists

\begin{tabular}{|l|l|}
\hline Complications & Number (\%) \\
\hline None & $65(68.4)$ \\
\hline Persistent pain & $10(10.5)$ \\
\hline Recurrent pain or instability & $5(5.2)$ \\
\hline Persistent painful instability & $3(3.2)$ \\
\hline Progressive degenerative disease & $5(5.2)$ \\
\hline Neuropraxia & $6(6.3)$ \\
\hline Neuroma & $1(1.0)$ \\
\hline Total & $30(31.6)$ \\
\hline
\end{tabular}

Table 8 ROM in patients undergoing revision surgery

\begin{tabular}{|l|l|l|l|l|l|l|}
\hline & \multicolumn{3}{|l|}{$\begin{array}{l}\text { Preoperative } \\
\text { ROM (degrees) }\end{array}$} & \multicolumn{3}{l|}{$\begin{array}{l}\text { Last clinical } \\
\text { follow-up, } \\
\text { ROM (degrees) }\end{array}$} \\
\hline & $\boldsymbol{n}$ & Mean & SD & $\boldsymbol{n}$ & Mean & SD \\
\hline Flexion & 9 & 47 & 18 & 7 & 46 & 16 \\
\hline Extension & 9 & 55 & 16 & 7 & 54 & 12 \\
\hline Radial deviation & 6 & 22 & 7 & 5 & 19 & 8 \\
\hline Ulnar deviation & 6 & 27 & 8 & 5 & 29 & 4 \\
\hline Pronation & 8 & 76 & 19 & 7 & 74 & 17 \\
\hline Supination & 8 & 60 & 29 & 7 & 51 & 28 \\
\hline $\begin{array}{l}\text { Grip strength } \\
\text { (kg) }\end{array}$ & 4 & 19 & 11 & 5 & 15 & 9 \\
\hline
\end{tabular}

Abbreviations: ROM, range of motion; SD, standard deviation.

ligaments when using tendon grafts, this reconstruction has demonstrated the ability to restore DRUJ kinematics biomechanically. ${ }^{17}$ This procedure has been our preferred method to restore stability and function of the DRUJ.

In their initial series, Adams and Berger reported symptomatic relief and stabilization of the DRUJ in 12 of 14 patients at a mean 2.2-year follow-up. ${ }^{1}$ Two patients had recurrent instability, one of which needed an additional stabilizing procedure. The other patient had an insufficient volar rim of the distal radius, contributing to instability of her DRUJ. This patient achieved sufficient and functional stability by wearing a brace that reduced the translation of the DRUJ in supination and pronation.

Seo et al described a series of 16 patients with posttraumatic unidirectional DRUJ instability who underwent the Adams-Berger procedure. ${ }^{14}$ At a mean 1.5-year follow-up, instability was resolved in 15 of the 16 patients, three of which had increased laxity but no joint translation. The mean MMWS improved in all but the one patient with recurrent subluxation, who required a sigmoid notch osteotomy. Teoh and Yam looked at nine patients who underwent anatomic DRUJ reconstruction at an average of 1.2 years short-term follow-up and 9 years long-term follow-up. ${ }^{18}$ All patients had improvement in grip strength and wrist scores. The wrist scores decreased from the short- to the long-term follow-up period. Only three of nine patients remained pain free at long-term follow-up and two patients had recurrent instability. ${ }^{18}$

Our study supports the current literature by demonstrating good outcomes after anatomic ligament reconstruction of the DRUJ with a $14 \%$ failure rate at a median 38.5 months follow-up. Grip strength was significantly increased, and ROM was unchanged except for a decrease in pronosupination, which is consistent with other reports. Although statistically significant, these changes were likely not clinically significant. Our data demonstrates a significantly increased rate of reconstructive failure in females compared to males and with the use of an interference screw for graft fixation. There were no differences between males and females and age, type of graft or graft fixation, follow-up, and time to revision. Furthermore, we did not find a difference in revision rate based on the age of the patient, time from injury, number of previous surgeries, sigmoid notch type, type of graft used, or involvement of dominant wrist. Based on the results of this study, we have abandoned the use of interference screws for this procedure and rely on suture anchors for initial tendon fixation and tensioning.

The long-term stability of the Adams-Berger reconstruction depends on solid tendon to bone tunnel healing. ${ }^{19}$ As the native enthesis is not regenerated in a surgical reconstruction, stability of tendon grafts in bone tunnels relies on new bone formation and collagen fiber integration. ${ }^{20-23}$ This is an important aspect for the surgeon to consider when planning the surgery and the postoperative rehabilitation to optimize the long-term outcome for the patient. Kalson et al biomechanically tested tendon graft fixation methods in the AdamsBerger procedure. ${ }^{24}$ They found that the maximum load to failure of the original suture method of tendon graft fixation was equal to bone anchor suture fixation, which were both superior to an inference screw. This mirrors our clinical results. This may be due to the difficulty in verifying tension using the interference screw, or due to the limited size of the bone tunnel that may not tolerate the wrist loads over time, leading to failure. The rate of procedural or mechanical failure was significantly higher with an interference screw compared to the loop method of graft fixation. We also feel that immobilization for 4 to 6 weeks in a long arm cast, followed by another 4 to 6 weeks in a removable Munster splint with cautious rehabilitation, is important for solid osseointegration of the tendon graft and restoration of DRUJ stability.

The current study is based on data from a tertiary level hospital, with a large sample size and moderate duration of follow-up. However, this is a retrospective study assessing outcomes for a single surgical procedure without a control group or comparison to other treatment modalities. Continued pain was not considered a failure of the procedure, as the indication for reconstruction was primarily DRUJ instability. In addition, we were unable to determine retrospectively if the continued pain a patient may experience was due to the DRUJ or other concomitant pathology. It is possible that some patients had persistent pain due to the DRUJ and did not want further surgery, despite having stability of their DRUJ, but we were unable to assess that 
scenario. To truly identify procedural success future, prospective studies must emphasize the use of validated subjective outcome scales at several time points during followup. Further limitations of our study are the incomplete records for the patient-reported questionnaires. There was limited power to detect variable associations with the risk of revision, as there were only 12 revisions identified; however, the study had $80 \%$ power to detect a hazard ratio of $\geq 5.1$.

The available literature on this topic consists of single center studies with limited sample number, limited time of follow-up, and outcome measurements often limited to functional outcome scores. This study provides the largest series to date with the longest reported clinical follow-up

The current study demonstrates approximately $13 \%$ revision rate in 93 patients/95 wrists at mean 65 months followup. An increased revision rate was associated with female gender when an interference screw was used to fixate the graft. Surgical revision was not associated with age at the time of surgery, chronicity of injury, type of graft used, sigmoid notch shape, involvement of the dominant wrist or number of surgeries prior to the Adams-Berger reconstruction.

\section{Note}

Institutional ethics was approved for the use of personal health data. All work was performed at the Mayo Clinic in Rochester, MN.

\section{Funding}

None.

\section{Conflict of Interest \\ None declared.}

\section{References}

1 Adams BD, Berger RA. An anatomic reconstruction of the distal radioulnar ligaments for posttraumatic distal radioulnar joint instability. J Hand Surg Am 2002;27(02):243-251

2 Adams BD, Lawler E. Chronic instability of the distal radioulnar joint. J Am Acad Orthop Surg 2007;15(09):571-575

3 Stuart PR, Berger RA, Linscheid RL, An KN. The dorsopalmar stability of the distal radioulnar joint. J Hand Surg Am 2000;25 (04):689-699

4 Atzei A. New trends in arthroscopic management of type 1-B TFCC injuries with DRUJ instability. J Hand Surg Eur Vol 2009;34(05): 582-591

5 Anderson ML, Larson AN, Moran SL, Cooney WP, Amrami KK, Berger RA. Clinical comparison of arthroscopic versus open repair of triangular fibrocartilage complex tears. J Hand Surg Am 2008; 33(05):675-682

6 Soreide E, Husby T, Haugstvedt JR. A long-term (20 years') followup after arthroscopically assisted repair of the TFCC. J Plast Surg Hand Surg 2017 Oct;51(05):296-300

7 Adams BD. Anatomic reconstruction of the distal radioulnar ligaments for DRUJ instability. Tech Hand Up Extrem Surg 2000;4(03):154-160

8 Kakar S, Carlsen BT, Moran SL, Berger RA. The management of chronic distal radioulnar instability. Hand Clin 2010;26(04):517-528

9 Zimmerman RM, Jupiter JB. Instability of the distal radioulnar joint. J Hand Surg Eur Vol 2014;39(07):727-738

10 Houdek MT, Wagner ER, Moran SL, Berger RA. Disorders of the distal radioulnar joint. Plast Reconstr Surg 2015;135(01):161-172

11 Tolat AR, Stanley JK, Trail IA. A cadaveric study of the anatomy and stability of the distal radioulnar joint in the coronal and transverse planes. J Hand Surg [Br] 1996;21(05):587-594

12 Johnston K, Durand D, Hildebrand KA. Chronic volar distal radioulnar joint instability: joint capsular plication to restore function. Can J Surg 2009;52(02):112-118

13 Stanley D, Herbert TJ. The Swanson ulnar head prosthesis for posttraumatic disorders of the distal radio-ulnar joint. J Hand Surg [Br] 1992;17(06):682-688

14 Seo KN, Park MJ, Kang HJ. Anatomic reconstruction of the distal radioulnar ligament for posttraumatic distal radioulnar joint instability. Clin Orthop Surg 2009;1(03):138-145

15 Lawler E, Adams BD. Reconstruction for DRUJ instability. Hand (N Y) 2007;2(03):123-126

16 Adams BD, Divelbiss BJ. Reconstruction of the posttraumatic unstable distal radioulnar joint. Orthop Clin North Am 2001;32 (02):353-363, x x.

17 Petersen MS, Adams BD. Biomechanical evaluation of distal radioulnar reconstructions. J Hand Surg Am 1993;18(02):328-334

18 Teoh LC, Yam AK. Anatomic reconstruction of the distal radioulnar ligaments: long-term results.J Hand Surg [Br] 2005;30(02):185-193

19 Ekdahl M, Wang JH, Ronga M, Fu FH. Graft healing in anterior cruciate ligament reconstruction. Knee Surg Sports Traumatol Arthrosc 2008;16(10):935-947

20 Thomopoulos S, Williams GR, Soslowsky LJ. Tendon to bone healing: differences in biomechanical, structural, and compositional properties due to a range of activity levels. J Biomech Eng 2003;125(01):106-113

21 Thomopoulos S, Genin GM, Galatz LM. The development and morphogenesis of the tendon-to-bone insertion - what development can teach us about healing -. J Musculoskelet Neuronal Interact 2010;10(01):35-45

22 Rodeo SA, Arnoczky SP, Torzilli PA, Hidaka C, Warren RF. Tendonhealing in a bone tunnel. A biomechanical and histological study in the dog. J Bone Joint Surg Am 1993;75(12):1795-1803

23 Gulotta LV, Rodeo SA. Biology of autograft and allograft healing in anterior cruciate ligament reconstruction. Clin Sports Med 2007; 26(04):509-524

24 Kalson NS, Charalambous CP, Powell ES, Hearnden A, Stanley JK. Tendon graft-ulna fixation in distal radio-ulnar joint stabilisation; biomechanical comparison of three graft-bone fixation methods. Hand (N Y) 2009;4(03):279-282 